\title{
Translation of In Vitro Transport Inhibition Studies to Clinical Drug-Drug Interactions for Glecaprevir and Pibrentasvirs
}

\author{
Matthew P. Kosloski, Daniel A.J. Bow, Ryota Kikuchi, Haoyu Wang, Elaine J. Kim, \\ Kennan Marsh, Federico Mensa, Jens Kort, and Wei Liu \\ AbbVie Inc., North Chicago, Illinois
}

Received February 6, 2019; accepted May 31, 2019

\begin{abstract}
Glecaprevir and pibrentasvir are oral direct-acting antiviral agents approved in combination for treatment of chronic hepatitis $C$ viral infection. In vitro studies identified the combination as potentially clinically relevant inhibitors of the efflux transporters $\mathrm{P}$-glycoprotein (P-gp), breast cancer resistance protein (BCRP), and the hepatic uptake transporters organic anion transporting polypeptide (OATP) 1B1 and OATP1B3. Glecaprevir inhibited P-gp, BCRP, OATP1B1, and OATP1B3 with $\mathrm{IC}_{50}$ values of $0.33,2.3,0.017$, and $0.064 \mu \mathrm{M}$, respectively. Pibrentasvir inhibited P-gp, BCRP, and OATP1B1 with $\mathrm{IC}_{50}$ values of $0.036,14$, and $1.3 \mu \mathrm{M}$, respectively. Neither agent inhibited organic cation transporter (OCT) 1, OCT2, organic anion transporter (OAT) 1, OAT3, multidrug and toxin extrusion (MATE) 1, or MATE2K. Openlabel phase 1 clinical drug-drug interaction studies were conducted in healthy subjects to evaluate interaction potential of glecaprevir/ pibrentasvir and coadministered selective substrates for P-gp
\end{abstract}

(digoxin, dabigatran etexilate, and sofosbuvir), BCRP (rosuvastatin and sofosbuvir), and OATP1B1/3 (pravastatin and rosuvastatin). The pharmacokinetic maximum plasma concentration $\left(C_{\max }\right)$ and area under the concentration-time curve (AUC) parameters were evaluated for probe substrates alone and in combination with glecaprevir/ pibrentasvir. The $C_{\max }$ central values increased by $72 \%, 105 \%$, $123 \%, 462 \%$, and $66 \%$ for digoxin, dabigatran, pravastatin, rosuvastatin, and sofosbuvir, respectively, and the AUC central values increased by $48 \%, 138 \%, 130 \%, 115 \%$, and $125 \%$ for digoxin, dabigatran, pravastatin, rosuvastatin, and sofosbuvir, respectively. Exposure of sofosbuvir metabolite GS-331007 (nucleoside analog) was similar with or without glecaprevir/pibrentasvir. The outcomes of the clinical drug-drug interaction studies confirmed clinically relevant inhibition of P-gp, BCRP, and OATP1B1/3, and were used to provide dosing guidance for the concomitant use of glecaprevir/pibrentasvir with relevant transporter substrates.

\section{Introduction}

The adoption of direct-acting antiviral agents (DAAs) has transformed the clinical treatment of chronic hepatitis $\mathrm{C}$ virus (HCV) infection. Long the standard of care, pegylated interferon and ribavirin have been replaced with all-oral DAA combination regimens offering increased efficacy, better tolerability and safety, and shorter treatment durations. These DAA regimens consist of two or more inhibitors of hepatitis $\mathrm{C}$ nonstructural protein (NS) 3/4A protease, NS5A, and NS5B polymerase and achieve sustained virological response rates of $\geq 95 \%$ across the six common HCV genotypes with as short as 8 weeks of treatment (Bourlière and Pietri, 2019). The

The study was sponsored by AbbVie Inc.

Glecaprevir is a nonstructural protein $3 / 4 \mathrm{~A}$ protease inhibitor identified by AbbVie Inc. and Enanta Pharmaceuticals, Inc., that has been coformulated with pibrentasvir, an NS5A inhibitor. AbbVie contributed to the study design, research, and interpretation of data, and the writing, reviewing, and approving of the publication. D.A.J.B., R.K., E.J.K., K.M., F.M., J.L., and W.L. are current employees of AbbVie. M.P.K. and H.W. are former AbbVie employees. All current and former AbbVie authors may hold AbbVie stock or stock options.

https://doi.org/10.1124/jpet.119.256966.

S This article has supplemental material available at jpet.aspetjournals.org. fixed-dose combination of glecaprevir (formerly ABT-493), a NS3/4A protease inhibitor, and pibrentasvir (formerly ABT-530), a NS5A inhibitor, is approved as a once-daily regimen for the treatment of chronic HCV infection with HCV genotypes 1-6 (Mavyret, 2019). Both agents exhibit a high barrier to development of resistance and maintain potent antiviral activity against common $\mathrm{HCV}$ variants that confer resistance to NS3/4A protease and NS5A inhibitors ( $\mathrm{Ng}$ et al., 2017, 2018). Glecaprevir and pibrentasvir are $97.5 \%$ and $>99.9 \%$ bound to plasma proteins, respectively (Gane et al., 2017; Mavyret, 2019). Metabolism by CYP3A plays a secondary role in the disposition of glecaprevir and $<1 \%$ of drug is recovered unchanged in the urine. Pibrentasvir is not metabolized or eliminated in urine.

Chronic HCV infection is frequently complicated by comorbid conditions (Louie et al., 2012) that require therapeutic interventions. Review of a real-world HCV-infected patient population found $31 \%-66 \%$ of the patients are at risk for potentially clinically significant drug-drug interactions between their concomitant medications and HCV DAA regimens containing a NS3/4A protease inhibitor and/or NS5B inhibitor

ABBREVIATIONS: AUC, area under the concentration-time curve; $\mathrm{AUC}_{0-24}$, area under the concentration-time curve from time 0 to 24 hours after dosing; BCRP, breast cancer resistance protein; BSA, bovine serum albumin; DAA, direct-acting antiviral agent; DDI, drug-drug interaction; HCV, hepatitis $C$ virus; $I_{\text {gut }}$, theoretical drug concentration in the gastrointestinal tract; $I_{\text {in,max }}$, estimated maximum plasma inhibitor concentration at the inlet to the liver; $I_{\max , \mathrm{u}}$, maximum unbound systemic plasma concentration of inhibitor; MATE, multidrug and toxin extrusion; NS, nonstructural protein; OAT, organic anion transporter; OATP, organic anion-transporting polypeptide; OCT, organic cation transporter; P-gp, P-glycoprotein. 
(Höner Zu Siederdissen et al., 2016). Current DAA regimens are often associated with clinically relevant inhibition of intestinal efflux and/or hepatic uptake drug transporters. Potential drug-drug interactions may be significant enough to require dose adjustment or use of alternative therapies, and particular attention must be given to the clinical management of such interactions (Back and Else, 2013; Serfaty, 2013).

$\mathrm{P}$-glycoprotein (P-gp) is an efflux transporter expressed in a variety of tissues including the apical membrane of intestinal epithelial cells, renal proximal tubular cells, brain capillary endothelial cells, and the canalicular membrane of hepatocytes (Giacomini et al., 2010). Efflux across the apical membrane of intestinal epithelium regulates oral bioavailability of many P-gp substrates, and due to high local drug concentrations in intestine following dissolution of oral dosage forms is a frequent site of P-gp-related drug-drug interactions. Digoxin (a cardiac glycoside), dabigatran etexilate (an oral direct thrombin inhibitor), and sofosbuvir (a HCV NS5B inhibitor) are all sensitive substrates of P-gp (Greiner et al., 1999; Härtter et al., 2012; Kirby et al., 2015); dabigatran etexilate is also selective for P-gp localized in the intestine due to rapid conversion to the non-P-gp substrate dabigatran following absorption (Chu et al., 2018). Breast cancer resistance protein (BCRP) is another efflux transporter with substantial overlap in localization and function to P-gp (Giacomini et al., 2010). No selective probes of BCRP activity are available for the evaluation of clinical drug-drug interaction potential, but rosuvastatin is often used as a probe for BCRP as well as the hepatic uptake transporters organic anion transporting polypeptide (OATP) 1B1 and OATP1B3 (Elsby et al., 2012). Sofosbuvir is also a substrate of BCRP in addition to P-gp. Cumulative evidence demonstrates the importance of OATP1B1/3 in the disposition of a variety of clinically used drugs-notably including statins (Giacomini et al., 2010). Pravastatin is a selective substrate of OATP1B1 and OATP1B3 (Elsby et al., 2012). In addition to utility as probe mechanistic substrates for P-gp, BCRP, and OATP transporters, clinical drug-drug interaction studies help formulate appropriate dosing recommendations to manage potential safety risks associated with elevated concentrations of studied drugs and other substrates of these transporters.

In vitro studies in membrane vesicles and transporter overexpressing cell lines were conducted to evaluate the inhibition potential of glecaprevir and pibrentasvir for clinically relevant transporters, namely, P-gp, BCRP, OATP1B1/3, organic cation transporter (OCT) 1, OCT2, organic anion transporter (OAT) 1, OAT3, multidrug and toxin extrusion (MATE) 1, and MATE2K. The results of in vitro studies were used to predict clinical drug-drug interaction (DDI) potential of glecaprevir/pibrentasvir, which were then confirmed in a series of clinical studies conducted in healthy volunteers with digoxin, dabigatran etexilate, sofosbuvir, rosuvastatin, and pravastatin.

\section{Materials and Methods}

\section{In Vitro Materials}

Glecaprevir and pibrentasvir were synthesized by AbbVie Inc. (North Chicago, IL); working stock solutions were prepared in dimethyl sulfoxide. All other materials were purchased from commercial sources unless stated otherwise.

\section{In Vitro Study Design}

Inhibition of P-gp and BCRP. Inhibition of P-gp and BCRP by glecaprevir or pibrentasvir was explored via evaluation of transport for known probe substrates in isolated membrane vesicles expressing the efflux transporter of interest. Transporter assay kits (P-gp and BCRP) were obtained from Solvo Biotechnology (Budapest, Hungary). Assays were conducted according to the kit instructions. P-gp and BCRP probes were $\left[{ }^{3} \mathrm{H}\right] \mathrm{N}$-methylquinidine and Lucifer yellow, respectively. The final concentration of the substrates and the incubation times are summarized in Supplemental Table 1. ATPdependent transport was calculated as the difference in values in the presence of AMP or ATP. Inhibition constants $\left(\mathrm{IC}_{50}\right)$ for glecaprevir or pibrentasvir were obtained by nonlinear least-squares fitting of $\log$ (inhibitor) versus normalized ATP-dependent transport of the reference substrate using a variable slope model in GraphPad Prism (La Jolla, CA). The $\mathrm{IC}_{50}$ values are presented as the geometric mean of two independent experiments.

Inhibition of OATP1B1/3, OCT1/2, OAT1/3, and MATE1/ 2K. Inhibition assessment for OATP1B1, OATP1B3, OCT1, OCT2, OAT1, OAT3, MATE1, and MATE2K was conducted as described previously (Kikuchi et al., 2017). The final concentration of the probe substrates and incubation times are summarized in Supplemental Table 1. The inhibitory effect of the test compound on the uptake of the reference substrates is presented as the percentage of inhibition of the control. The $\mathrm{IC}_{50}$ values were determined assuming competitive inhibition in GraphPad Prism. Owing to excessive binding of pibrentasvir to plasma proteins, inhibition studies were conducted in the presence of $4 \%$ weight per volume of bovine serum albumin (BSA) to determine transporter inhibition under semiphysiologic conditions in addition to the conventional protein-free condition. The $\mathrm{IC}_{50}$ values are presented as the geometric mean of two independent experiments.

Prediction of Transporter Inhibition. Predictions of clinical drug-drug interaction with transporters were made according to the Food and Drug Administration's guidance on in vitro metabolism- and transporter- mediated DDI studies (https://www.fda.gov/downloads/ Drugs/Guidances/UCM581965.pdf). The pharmacokinetic parameters used in the calculation of the interaction potential are listed in Supplemental Table 2. For the prediction of P-gp and BCRP inhibition, $I_{\text {gut }} / \mathrm{IC}_{50}>10$ was considered potentially clinically relevant, where $I_{\text {gut }}$ is the theoretical drug concentration in the gastrointestinal tract (dose $/ 250 \mathrm{ml}$ ). The $R$-free and $R$-total values for the prediction of in vivo OATP1B1, OATP1B3, and OCT1 inhibition were calculated using the $\mathrm{IC}_{50}$ values obtained in the absence of BSA ( $R$-free) or presence of $4 \%$ BSA ( $R$-total) by the following equations (Kikuchi et al., 2017):

$$
\begin{gathered}
R-\text { free }=1+\frac{f_{\mathrm{u}, \mathrm{p}} \times I_{\text {in,max }}}{\mathrm{IC}_{50}} \\
R-\text { total }=1+\frac{I_{\text {in,max }}}{\mathrm{IC}_{50, \text { total }}} \\
I_{\text {in,max }}=I_{\text {max }}+\frac{F_{a} F_{g} \times k_{a} \times \text { Dose }}{Q_{\mathrm{h}} \times R_{\mathrm{B}}}
\end{gathered}
$$

where $f_{\mathrm{u}, \mathrm{p}}$ is the fraction of unbound inhibitor in plasma; $I_{\mathrm{in} \text {,max }}$ is the estimated maximum plasma inhibitor concentration at the inlet to the liver; $I_{\max }$ is the maximum systemic plasma concentration of inhibitor; $F_{a} F_{g}$ is the fraction of the dose of inhibitor that is absorbed; $k_{a}$ is the absorption rate constant of the inhibitor; Dose is the inhibitor dose; $Q_{\mathrm{h}}$ is the hepatic blood flow ( $97 \mathrm{~L} / \mathrm{h}$ per $70 \mathrm{~kg}$ ); and $R_{\mathrm{B}}$ is the blood-toplasma concentration ratio. Values of $R \geq 1.1$ were considered potentially clinically relevant (https://www.fda.gov/downloads/Drugs/ Guidances/UCM581965.pdf).

For the prediction of OCT2, OAT1, OAT3, MATE1, and MATE2K inhibition, $I_{\max , \mathrm{u}} / \mathrm{IC}_{50}$ values were calculated, where $I_{\max , \mathrm{u}}$ is the maximum unbound systemic plasma concentration of inhibitor; $I_{\max , \mathrm{u}} /$ $\mathrm{IC}_{50}>0.1$ (OCT2, OAT1, and OAT3) and $I_{\max , \mathrm{u}} / \mathrm{IC}_{50}>0.02$ (MATE1 and MATE2K) were considered potentially clinically relevant. 


\section{Clinical Study Design}

Study Participants and Designs. Open-label, phase 1 clinical studies were conducted under Good Clinical Practice Guidelines and ethical principles that have their origin in the Declaration of Helsinki. All studies were conducted at the AbbVie Clinical Pharmacology Research Unit. Study protocols and amendments were approved by the Vista Health System institutional review boards (Waukegan, IL). A signed informed consent was obtained from each subject.

Adult male and female subjects between the ages of 18 and 55 years, with a body mass index between 18 and $30 \mathrm{~kg} / \mathrm{m}^{2}$ and in general good health, were enrolled. Female subjects were of nonchildbearing potential. The use of medications that might have inhibited or induced metabolic enzymes and drug transporters of interest was prohibited 1 month prior to dosing of study drugs through study completion. Twelve subjects participated in each of the digoxin, dabigatran etexilate, pravastatin, and rosuvastatin studies and 16 subjects participated in the sofosbuvir study. The study design schematics are depicted in Fig. 1. All study drugs were administered orally in the morning 30 minutes after a standardized breakfast each day.

Digoxin study. Single doses of digoxin $0.5 \mathrm{mg}$ were administered in period 1 on day 1 and in period 2 on day 8 . Glecaprevir $400 \mathrm{mg}$ and pibrentasvir $120 \mathrm{mg}$ were administered once a day in period 2 on days $1-12$. The first dose in periods 1 and 2 was separated by a 10-day washout. Blood samples were collected for pharmacokinetic assessment of digoxin plasma concentrations up to 120 hours after doses in period 1 on day 1 (alone) and period 2 on day 8 (coadministered) and for glecaprevir and pibrentasvir plasma concentrations up to 24 hours after doses in period 2 on day 7 (alone) and day 8 (coadministered). Urine was collected for digoxin analysis up to 120 hours after doses in period 1 on day 1 and period 2 on day 8 .

Dabigatran etexilate study. Single doses of dabigatran etexilate mesylate (dabigatran etexilate $150 \mathrm{mg}$ equivalent) were administered on days 1 and 11 . Glecaprevir $300 \mathrm{mg}$ and pibrentasvir $120 \mathrm{mg}$ were administered once a day on days 4-13. Blood samples were collected for pharmacokinetic assessment of dabigatran plasma concentrations up to 72 hours after doses on day 1 (alone) and day 11 (coadministered) and for glecaprevir and pibrentasvir plasma concentrations up to 24 hours after doses on day 10 (alone) and day 11 (coadministered).

Pravastatin study. Multiple doses of pravastatin $10 \mathrm{mg}$ once a day were administered in period 2 on days $1-3$ and period 3 on days 1-7. Glecaprevir $400 \mathrm{mg}$ and pibrentasvir $120 \mathrm{mg}$ were administered as a single dose in period 1 on day 1 and once a day in period 3 on days $1-7$. The first doses in periods 1 and 2 were separated by an 8-day washout interval. No washout interval separated periods 2 and 3 . Blood samples were collected for pharmacokinetic assessment of pravastatin plasma concentrations up to 24 hours after doses in period 2 on day 3 (alone) and period 3 on day 7 (coadministered) and for glecaprevir and pibrentasvir plasma concentrations up to 24 hours after doses in period 1 on day 1 (alone) and period 3 on day 1 (coadministered).

Rosuvastatin study. Multiple doses of rosuvastatin $5 \mathrm{mg}$ once a day were administered in period 2 on days $1-7$ and period 3 on days 1-7. Glecaprevir $400 \mathrm{mg}$ and pibrentasvir $120 \mathrm{mg}$ were administered as a single dose in period 1 on day 1 and once a day in period 3 on days $1-7$. The first dose in periods 1 and 2 was separated by an 8-day washout interval. No washout interval separated periods 2 and 3. Blood samples were collected for pharmacokinetic assessment of rosuvastatin plasma concentrations up to 24 hours after doses in period 2 on day 7 (alone) and period 3 on day 7 (coadministered) and for glecaprevir and pibrentasvir plasma concentrations up to 24 hours after doses in period 1 on day 1 (alone) and period 3 on day 1 (coadministered).

Sofosbuvir study. This study consisted of two cohorts. In cohort 1 , multiple doses of glecaprevir $400 \mathrm{mg}$ and pibrentasvir $120 \mathrm{mg}$ once a day were administered on days 1-7 of period 1 and then sofosbuvir $400 \mathrm{mg}$ was administered with glecaprevir $400 \mathrm{mg}$ and pibrentasvir $120 \mathrm{mg}$ once a day on days $1-7$ of period 2 . In cohort 2 , multiple doses of sofosbuvir $400 \mathrm{mg}$ once a day were administered on days $1-7$ of period 1 and then sofosbuvir $400 \mathrm{mg}$ was administered with glecaprevir $400 \mathrm{mg}$ and pibrentasvir $120 \mathrm{mg}$ once a day on days $1-7$ of period 2 . Blood samples were collected for pharmacokinetic assessment of sofosbuvir and GS-331007 (nucleoside analog) plasma concentrations at up to 24 hours after dosing in period 1 on day 7 (alone) and period 2
Single-dose substrate design (digoxin)

\begin{tabular}{|c|c|c|c|c|}
\hline Period 1 & \multirow{4}{*}{ Washout } & \multicolumn{3}{|c|}{ Period 2} \\
\hline Day 1 & & Days 1.7 & Day 8 & Days $9-12$ \\
\hline \multirow[t]{2}{*}{ Digoxin } & & & Digoxin & \\
\hline & & \multicolumn{3}{|c|}{ Glecaprevir + Pibrentasvir QD } \\
\hline
\end{tabular}

Single-dose substrate design (dabigatran)

\begin{tabular}{|c|c|c|c|c|}
\hline Day 1 & Days 2 to 3 & Days 4 to 10 & Day 11 & Days 12 to 13 \\
\hline Dabigatran etexilate & & & Dabigatran etexilate & \\
\hline & & \multicolumn{3}{|c|}{ Glecaprevir + Pibrentasvir QD } \\
\hline
\end{tabular}

Multiple-dose substrate design (pravastatin, rosuvastatin)

\begin{tabular}{|c|c|c|c|}
\hline Period 1 & \multirow{4}{*}{ Washout } & Period 2 & Period 3 \\
\hline \multirow[t]{2}{*}{ Day 1} & & Days 1 to 3 or $7^{3}$ & Days 1 to 7 \\
\hline & & \multicolumn{2}{|c|}{ Pravastatin $Q D$ or Rosuvastatin $Q D$} \\
\hline Glecaprevir + Pibr & & & evir + Pibrentasvir QD \\
\hline
\end{tabular}

Multiple-dose substrate design (sofosbuvir)

\begin{tabular}{|c|c|c|}
\hline Cohort & Period 1 (Days 1 to 7 ) & Period 2 (Days 1 to 7 ) \\
\hline \multirow{2}{*}{1} & & Sofosbuvir $Q D$ \\
\hline & \multicolumn{2}{|c|}{ Glecaprevir + Pibrentasvir QD } \\
\hline \multirow{2}{*}{2} & \multicolumn{2}{|c|}{ Sofosbuvir QD } \\
\hline & & caprevir + Pibrent \\
\hline
\end{tabular}

a. Doses were administered in Period 2 for 3 days (pravastatin) or 7 days (rosuvastatin)

$\mathrm{QD}=$ once daily
Fig. 1. Clinical DDI study designs used to evaluate interactions of glecaprevir and pibrentasvir with digoxin, dabigatran etexilate, pravastatin, rosuvastatin, and sofosbuvir. 
on day 7 (coadministered) in cohort 2 and for glecaprevir and pibrentasvir plasma concentrations up to 24 hours after doses in period 1 on day 7 (alone) and period 2 on day 7 (combination) in cohort 1.

Sample Analysis. Plasma concentrations of all drugs were determined using validated liquid chromatography methods with tandem mass spectrometric detection. The lower limit of quantitation in plasma was established at $\leq 0.205 \mathrm{ng} / \mathrm{ml}$ (glecaprevir and pibrentasvir across studies), $1.00 \mathrm{ng} / \mathrm{ml}$ (total dabigatran), $0.0100 \mathrm{ng} / \mathrm{ml}$ (digoxin), $0.500 \mathrm{ng} / \mathrm{ml}$ (pravastatin), $0.100 \mathrm{ng} / \mathrm{ml}$ (rosuvastatin), $5 \mathrm{ng} / \mathrm{ml}$ (sofosbuvir), and $10 \mathrm{ng} / \mathrm{ml}$ (GS-331007). The lower limit of quantitation for digoxin in urine was $2.00 \mathrm{ng} / \mathrm{ml}$. Drug concentrations below the respective lower limit of quantitation were reported as zero. A summary of the validated analytical methods is provided in Supplemental Table 3.

Pharmacokinetic and Statistical Analyses. For each analyte, where applicable, the maximum observed plasma concentration $\left(C_{\max }\right)$, the time to $C_{\max }$, the predose trough concentration, and the trough concentration 24 hours after dosing were determined directly from the concentration-time data. Pharmacokinetic parameters including the area under the concentration-time curve (AUC) from time 0 to 24 hours after dosing $\left(\mathrm{AUC}_{0-24}\right)$, AUC from time 0 to the last measurable concentration, AUC from time 0 to infinity, terminal elimination half-life in plasma, the fraction of unchanged drug excreted in urine, and renal clearance were calculated via noncompartmental methods.

Pharmacokinetic and statistical analyses were conducted using SAS version 9.2 (SAS Institute Inc., Cary, NC). To assess the effect of coadministration with glecaprevir and pibrentasvir on each of the interacting medications, a repeated measures analysis was performed for the natural logarithms of the $C_{\max }$, AUC, and/or the trough concentration 24 hours after dosing. Central value ratios and $90 \%$ confidence intervals were calculated. Repeated measures analyses were performed to assess the effect of coadministration with substrate drugs on glecaprevir and pibrentasvir $C_{\max }, \mathrm{AUC}_{0-24}$, and the trough concentration 24 hours after dosing. Pharmacokinetic parameters were considered similar with and without concomitant drugs if central value ratios were $\geq 0.80$ and $\leq 1.25$.

Safety and Tolerability Assessments. Safety and tolerability throughout each study were assessed via adverse event monitoring, laboratory tests, and vital signs measurements.

\section{Results}

\section{In Vitro Study Results}

In Vitro Inhibition of Drug Transporters. In vitro $\mathrm{IC}_{50}$ values for glecaprevir and pibrentasvir and the associated inhibition profiles are given in Table 1 and shown in Supplemental Fig. 1. Glecaprevir inhibited P-gp, BCRP, OATP1B1, and OATP1B3 with $\mathrm{IC}_{50}$ values of $0.33,2.3$, 0.017 , and $0.064 \mu \mathrm{M}$ respectively. Glecaprevir showed less than $50 \%$ inhibition of OCT2, OAT1, and OAT3 at $100 \mu \mathrm{M}$ and less than 50\% inhibition of OCT1, MATE1, and MATE2K at $30 \mu \mathrm{M} ; \mathrm{IC}_{50}$ values for these transporters are considered as $>100 \mu \mathrm{M}$ (OCT2, OAT1, and OAT3) and >30 $\mu \mathrm{M}$ (OCT1, MATE1, and MATE2K). Pibrentasvir inhibited P-gp and BCRP with $\mathrm{IC}_{50}$ values of 0.036 and $14 \mu \mathrm{M}$, respectively. Pibrentasvir inhibited OATP1B1 in the absence of BSA in a concentration-dependent manner up to $3 \mu \mathrm{M}$; however, further inhibition was not observed at higher concentrations (30 and $300 \mu \mathrm{M}$ ). An $\mathrm{IC}_{50}$ value of $1.3 \mu \mathrm{M}$ for pibrentasvir against OATP1B1 was determined when the assay was conducted in the presence of $4 \%$ BSA to mimic physiologic conditions. Pibrentasvir showed less than 50\% inhibition at the concentrations tested ( 3 and $30 \mu \mathrm{M}$ ) for OATP1B3, OCT1, OCT2, OAT1, OAT3, MATE1, and MATE2K in the absence and presence of $4 \% \mathrm{BSA} ; \mathrm{IC}_{50}$ values for these transporters are considered to be $>30 \mu \mathrm{M}$.

Prediction of Transporter Inhibition. Inhibition of intestinal efflux transporters (P-gp and BCRP) was predicted based on the theoretical concentrations (dose $/ 250 \mathrm{ml}$ ) for both glecaprevir and pibrentasvir in the gastrointestinal tract: values of $I_{\text {gut }} / \mathrm{IC}_{50}>10$ (Table 1). Using anticipated exposure of glecaprevir for the glecaprevir/pibrentasvir 300/120 mg dose combination in HCV-infected subjects, unbound drug exposure in the hepatic portal vein was estimated to be sufficient to inhibit OATP1B1 and OATP1B3: values of $R \geq$ 1.1 (Table 1). As for pibrentasvir, inhibition of OATP1B1, but not OATP1B3, was predicted using the $\mathrm{IC}_{50}$ value determined in the presence of $4 \% \mathrm{BSA}: R$-total $=1.4$ (Table 1$)$. Glecaprevir and pibrentasvir are not predicted to inhibit OCT1, OCT2, OAT1, OAT3, MATE1, and MATE2K.

\section{Clinical Study Results}

Subject Demographics and Disposition. Sixty-four subjects received at least one dose of the study drugs across the studies. Of these 64 subjects, $83 \%$ (53/64) were male, $55 \%$ (35/64) were white, $42 \%$ (27/64) were black, and 5\% (4/76) were of other races. Subjects ranged from 19 to 54 years of age and from 50 to $107 \mathrm{~kg}$ in body weight. Subjects enrolled in the

TABLE 1

In vitro inhibition of clinically relevant transporters

Glecaprevir: $I_{\text {gut }}=1430 \mu \mathrm{M}, I_{\text {in, } \max }=3.0 \mu \mathrm{M}$, and $I_{\text {max }, \mathrm{u}}=0.018 \mu \mathrm{M}$; Pibrentasvir: $I_{\text {gut }}=431 \mu \mathrm{M}, I_{\text {in, } \max }=0.56 \mu \mathrm{M}$, and $I_{\max , \mathrm{u}}=0.00099 \mu \mathrm{M}$

\begin{tabular}{|c|c|c|c|c|c|c|c|c|}
\hline \multirow{2}{*}{ Transporter } & \multicolumn{4}{|c|}{ Glecaprevir } & \multicolumn{4}{|c|}{ Pibrentasvir } \\
\hline & $\mathrm{IC}_{50}$ & $I_{\text {gut }} / \mathrm{IC}_{50}$ & $R$ value & $I_{\max , \mathrm{u}} / \mathrm{IC}_{50}$ & $\mathrm{IC}_{50}$ & $I_{\text {gut }} / \mathrm{IC}_{50}$ & $R$ value & $I_{\max , \mathrm{u}} / \mathrm{IC}_{50}$ \\
\hline & $\mu M$ & & & & $\mu M$ & & & \\
\hline P-gp & 0.33 & 4335 & - & - & 0.036 & 11,978 & - & - \\
\hline BCRP & 2.3 & 622 & - & - & 14 & 31 & - & - \\
\hline OATP1B1 & 0.017 & - & 5.5 & - & $1.3^{a}$ & - & $1.4^{b}$ & - \\
\hline OATP1B3 & 0.064 & - & 2.2 & - & $>30$ & - & $<1.1$ & - \\
\hline OCT1 & $>30$ & - & $<1.1$ & - & $>30$ & - & $<1.1$ & - \\
\hline OCT2 & $>100$ & - & - & $<0.1$ & $>30$ & - & - & $<0.1$ \\
\hline OAT1 & $>100$ & - & - & $<0.1$ & $>30$ & - & - & $<0.1$ \\
\hline OAT3 & $>100$ & - & - & $<0.1$ & $>30$ & - & - & $<0.1$ \\
\hline MATE1 & $>30$ & - & - & $<0.02$ & $>30$ & - & - & $<0.02$ \\
\hline MATE2K & $>30$ & - & - & $<0.02$ & $>30$ & - & - & $<0.02$ \\
\hline
\end{tabular}

-, not applicable.

${ }^{a}$ Value determined in the inhibition studies in $4 \%$ BSA.

${ }^{b} R$-total value. 
digoxin, pravastatin, and sofosbuvir studies completed all study activities. One subject who withdrew consent in the dabigatran study and one subject who discontinued the rosuvastatin study after a panic attack were excluded from pharmacokinetic analyses. One subject discontinued the study drug in the dabigatran study prior to receiving the final dose of glecaprevir and pibrentasvir, but was included in all analyses.

Pharmacokinetics. Complete pharmacokinetic parameter data for glecaprevir, pibrentasvir, probe substrates, and coadministered drugs when administered alone or in combination are given in Table 2 and discussed subsequently. Concentration-time profiles in plasma are presented for probe substrates in Fig. 2 and for glecaprevir and pibrentasvir in Fig. 3. Central value ratios for the probe substrates when administered with glecaprevir and pibrentasvir relative to administration alone are summarized in Fig. 4.

Digoxin ( $P$-gp). The $C_{\max }$ and AUC from time 0 to infinity central values for the P-gp substrate digoxin were $72 \%$ and $48 \%$ higher, respectively, when digoxin was coadministered with glecaprevir and pibrentasvir than for digoxin alone. The digoxin mean fraction excreted in urine increased from $57.8 \%$ to $68.1 \%$ and the mean renal clearance decreased from 9.68 to $6.99 \mathrm{~L} / \mathrm{h}$ during coadministration. The glecaprevir and pibrentasvir $C_{\text {max }}$ and $\mathrm{AUC}_{0-24}$ central values were similar with or without digoxin ( $\leq 16 \%$ difference).

Dabigatran etexilate ( $P$-gp). Dabigatran etexilate is a prodrug for the pharmacologically active moiety dabigatran. When the P-gp substrate dabigatran etexilate was coadministered with glecaprevir and pibrentasvir, dabigatran $C_{\max }$ and AUC from time 0 to infinity central values were $105 \%$ and $138 \%$ higher, respectively, compared with when dabigatran etexilate was administered alone. The glecaprevir and pibrentasvir $C_{\max }$ and $\mathrm{AUC}_{0-24}$ central values were similar with or without dabigatran etexilate ( $\leq 16 \%$ difference).

Pravastatin (OATP1B1/3). The $C_{\max }$ and $\mathrm{AUC}_{0-24}$ central values of pravastatin, a substrate of OATP1B1/3, were $123 \%$ and $130 \%$ higher, respectively, when coadministered with multiple doses of glecaprevir and pibrenatasvir compared with when pravastatin was administered alone. When coadministered with multiple doses of pravastatin, the first dose of glecaprevir and pibrentasvir exhibited $59 \%$ and $44 \%$ higher glecaprevir $C_{\max }$ and $\mathrm{AUC}_{0-24}$ values, respectively, but pibrentasvir exposure was similar ( $\leq 24 \%$ difference) to when glecaprevir and pibrentasvir were administered alone as a single dose.

Rosuvastatin (OATP1B1/3 and BCRP). Following coadministration of rosuvastatin, a substrate of OATP1B1/3 and
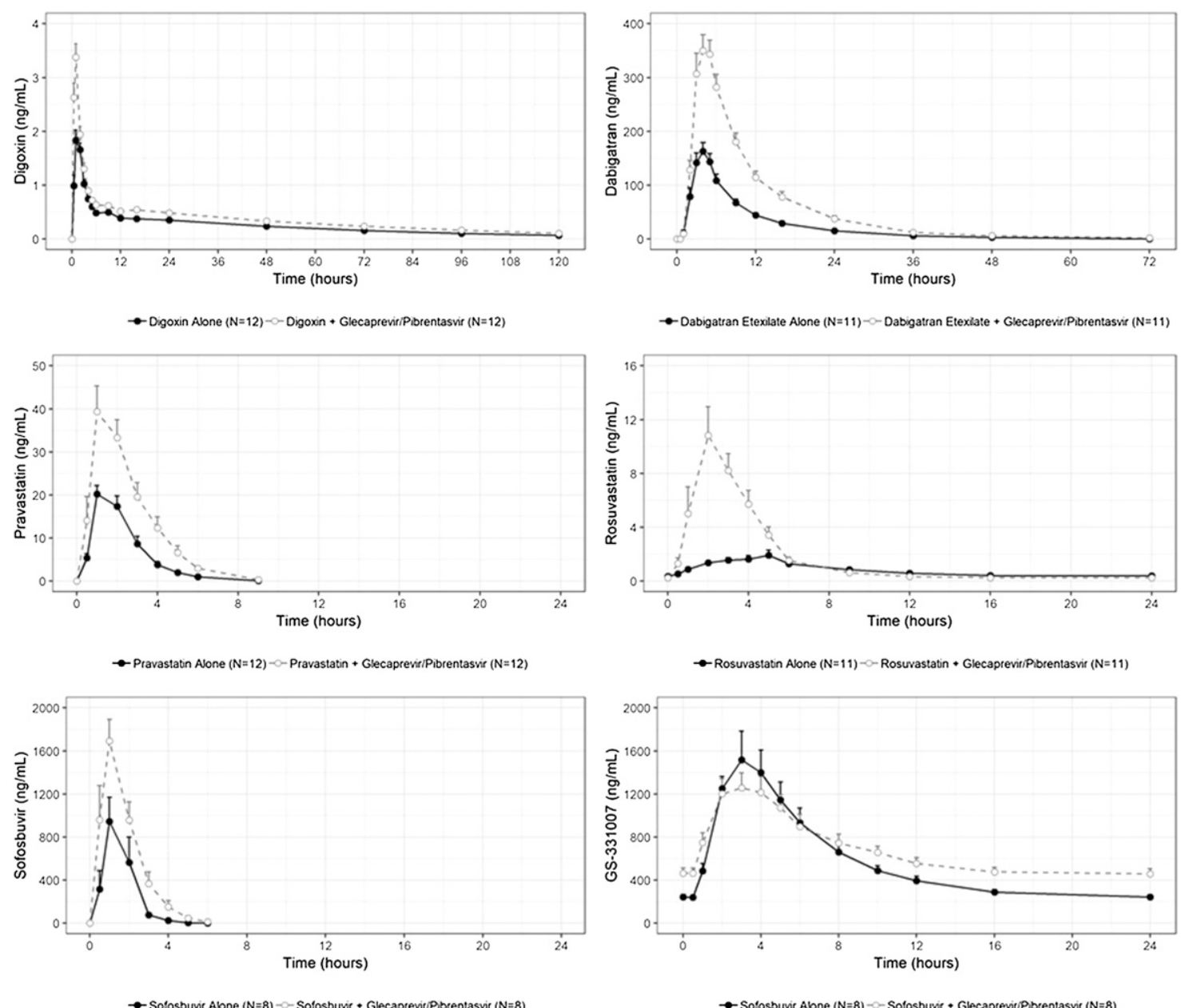

Fig. 2. Plasma concentration-time profiles of probe substrates administered alone (closed circles) or with glecaprevir/pibrentasvir (open circles) in healthy subjects. Data are presented as mean + S.E. 


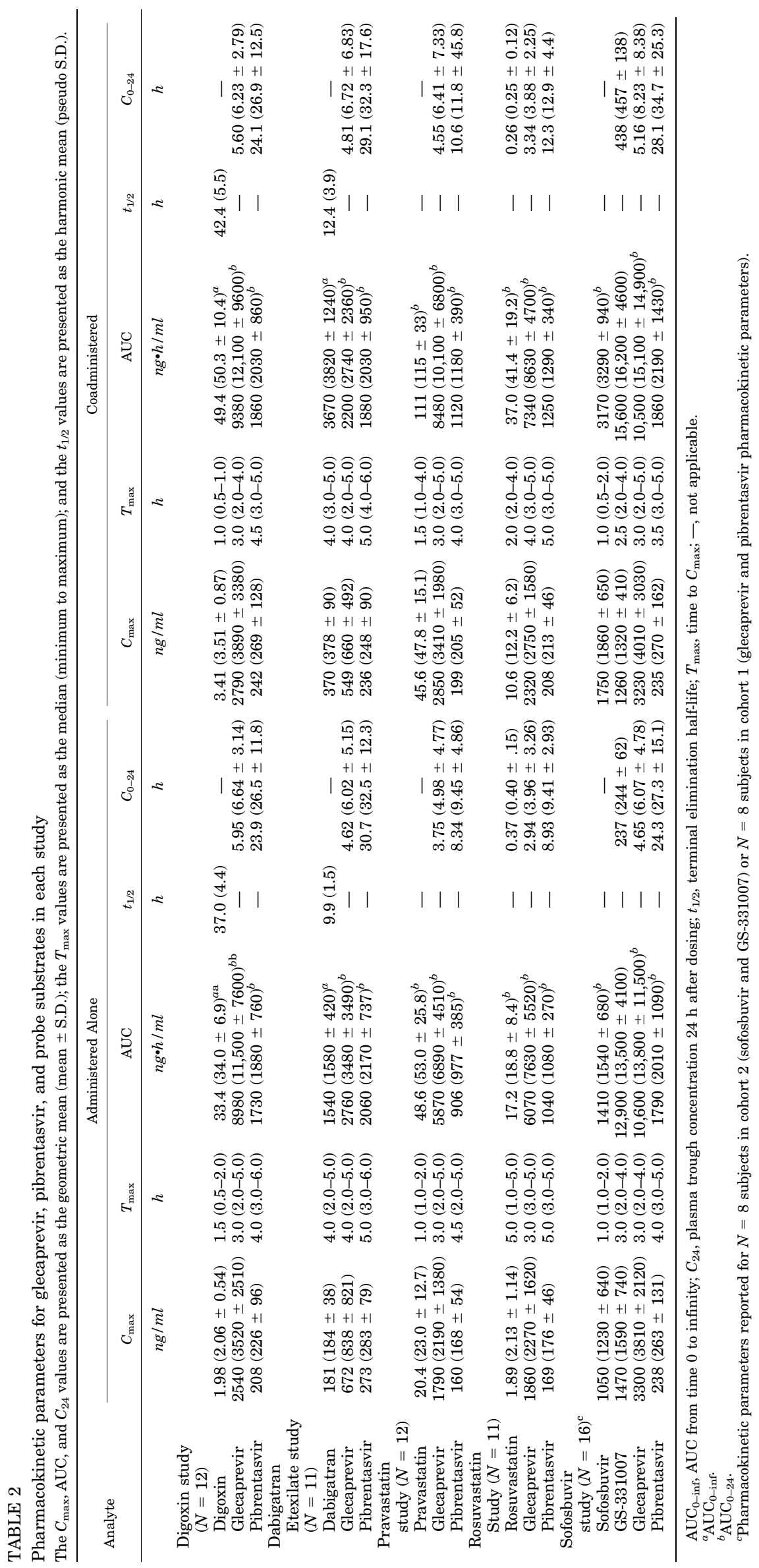



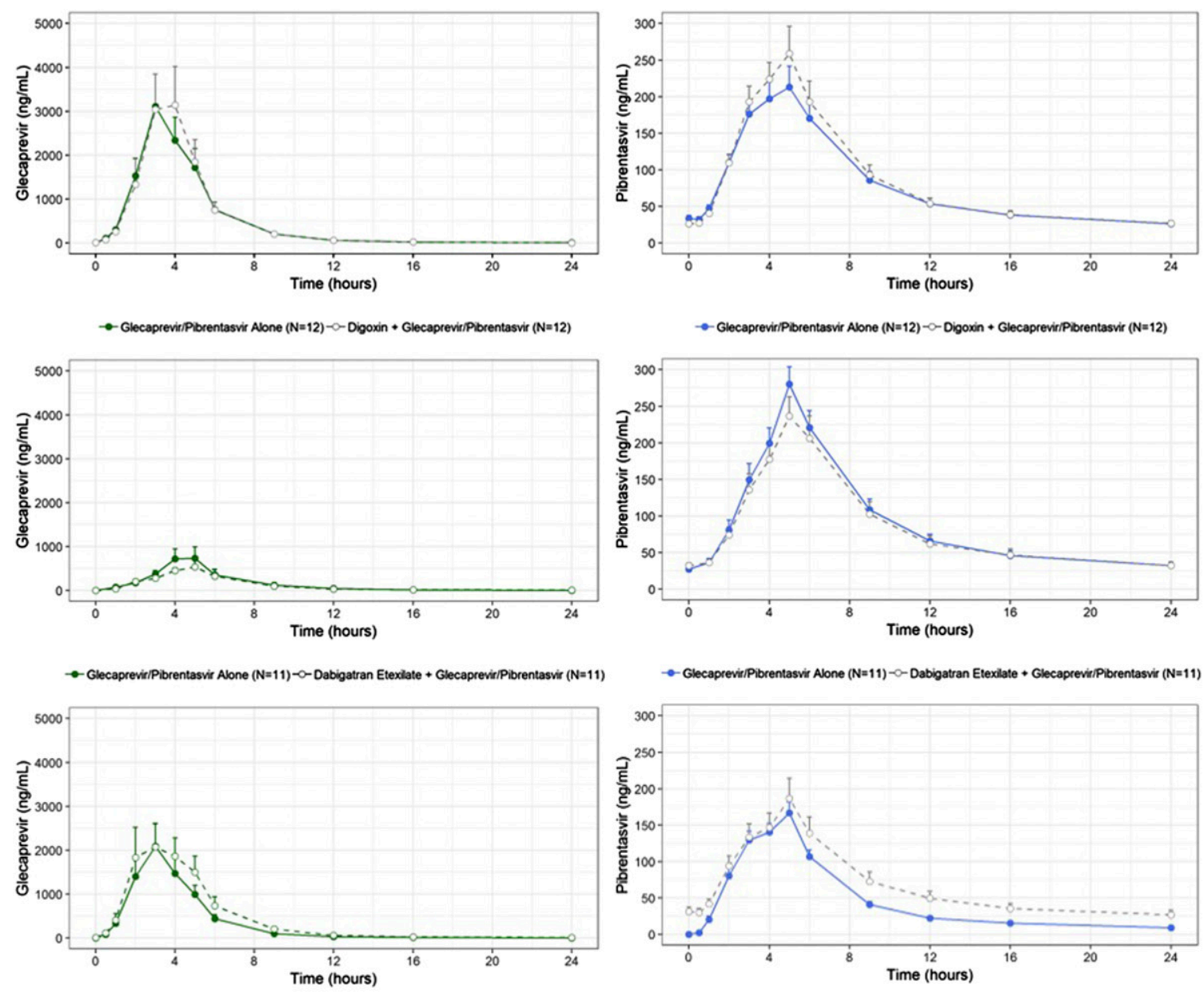

$\rightarrow-$ Glecaprevir/Pibrentasvir Alone (N=11) $-0-$ Rosuvastatin * GlecapreviriPlibentasvir $(\mathrm{N}=11)$
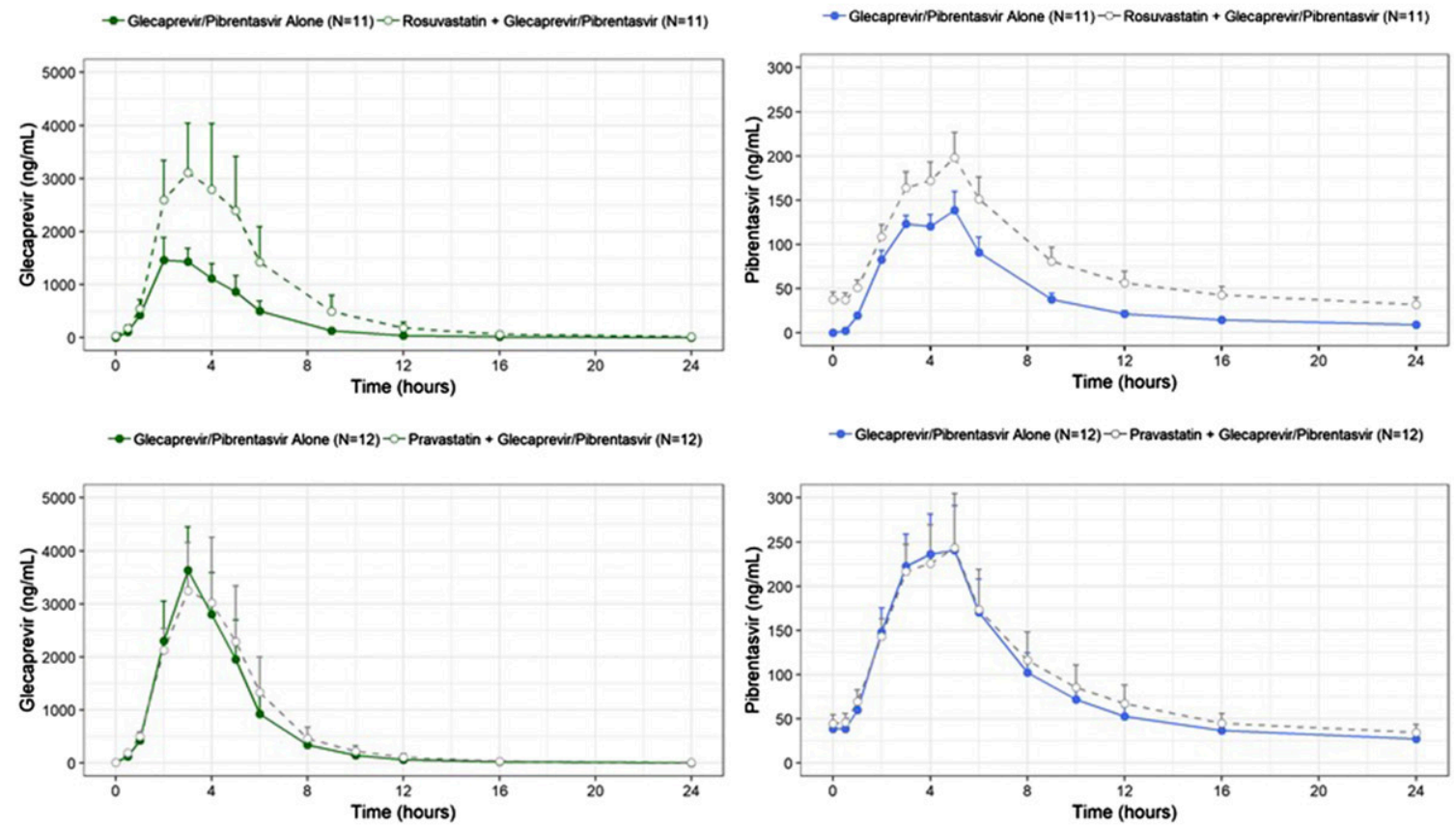

$\rightarrow-$ GlecapreviriPibrentasvir Alone (N=12) $\sim-$ Pravastatin + GlecapreviriPibrentasvir $(\mathrm{N}=12)$

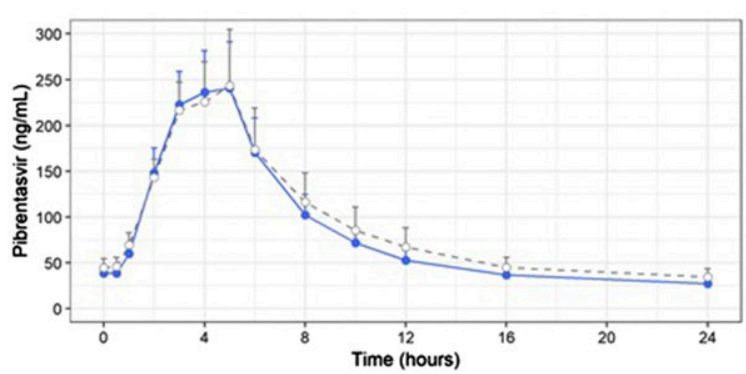

- Glecaprevitipibrentasvit Alone $(\mathrm{N}=8)-\infty$ Sofosbuvir + GlecapreviriPibrentasvir $(\mathrm{N}=8)$

- GlecapreviriPibrentasvir Alone (N=8) - - Sofosbuvir * Glecaprevir/Pibrentasvir $(\mathrm{N}=8)$

Fig. 3. Plasma concentration-time profiles of glecaprevir (blue) and pibrentasvir (green) when administered alone (closed circles) or with probe substrates (open circles) in healthy subjects. Data are presented as mean + S.E. 


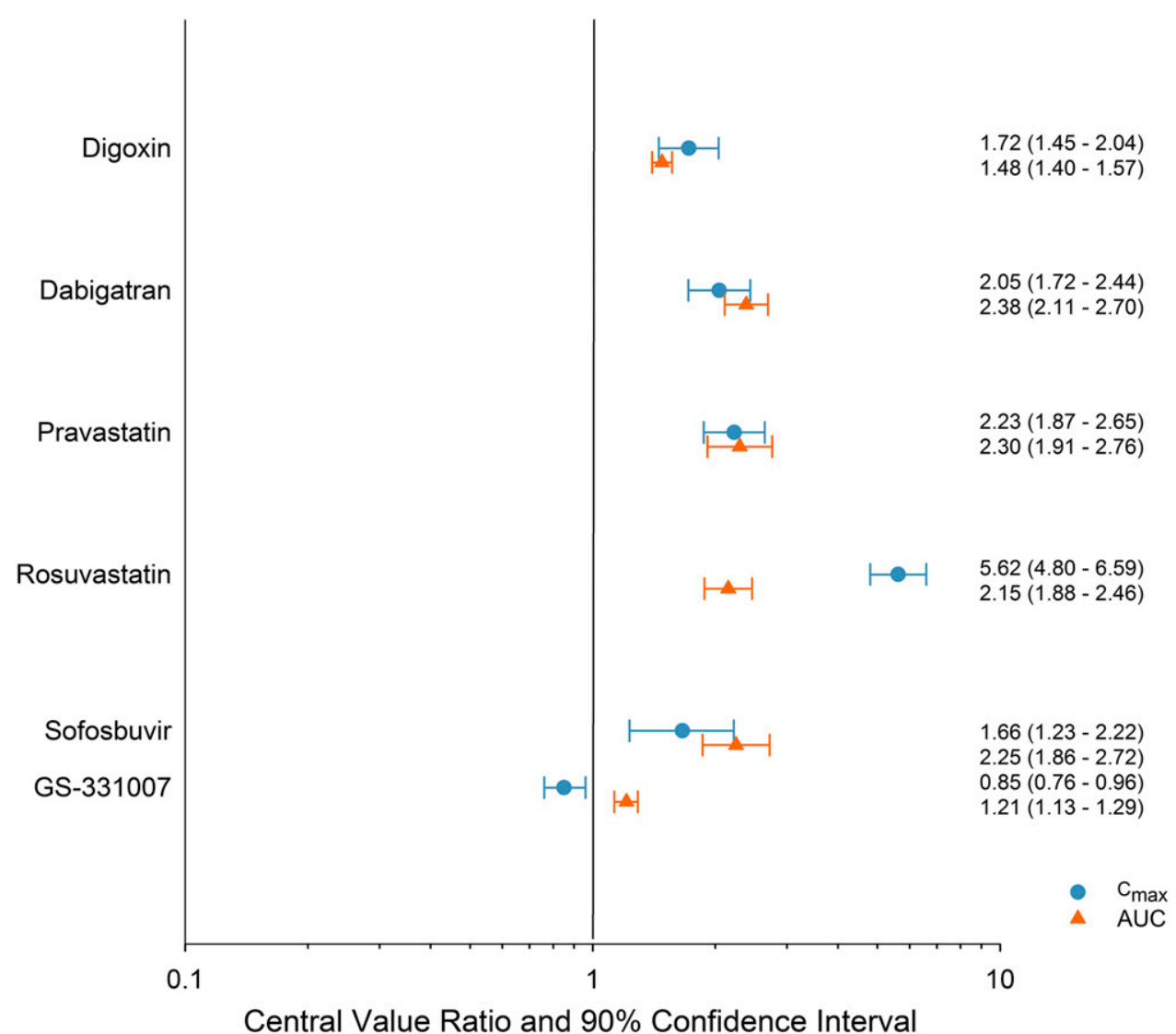

Fig. 4. Central value ratios and $90 \%$ confidence intervals for the effect of glecaprevir and pibrentasvir on concomitant medications.
BCRP, with glecaprevir and pibrentasvir, the rosuvastatin $C_{\text {max }}$ and $\mathrm{AUC}_{0-24}$ central values were $462 \%$ and $115 \%$ higher, respectively, compared with when rosuvastatin was administered alone. Similar exposures were observed when the first glecaprevir and pibrentasvir dose was administered with rosuvastatin and when glecaprevir and pibrentasvir were administered alone ( $\leq 25 \%$ difference).

Sofosbuvir (P-gp and BCRP). Following coadministration of sofosbuvir, a substrate of P-gp and BCRP, with glecaprevir and pibrentasvir, the sofosbuvir $C_{\max }$ and $\mathrm{AUC}_{0-24}$ central values were $66 \%$ and $125 \%$ higher, respectively, compared with when sofosbuvir was administered alone; sofosbuvir metabolite GS$331007 C_{\max }$ and $\mathrm{AUC}_{0-24}$ central values were similar to without glecaprevir and pibrentasvir ( $\leq 21 \%$ difference). Steady-state exposures of glecaprevir and pibrentasvir with sofosbuvir were minimally affected ( $\leq 16 \%$ change in central values) compared with administration of glecaprevir and pibrentasvir alone.

Safety. No serious adverse events were reported in any of the studies. One subject in the dabigatran etexilate study and one subject in the rosuvastatin study discontinued study drug due to adverse events that were assessed by the investigator as having no reasonable possibility of being related to the study drugs. No clinically significant vital signs, laboratory values, or electrocardiogram measurements were observed during the course of the studies.

\section{Discussion}

Direct-acting antiviral regimens containing NS3/4A protease inhibitors (e.g., paritaprevir, grazoprevir, and voxilaprevir) or
NS5A inhibitors (e.g., ledipasvir, velpatasvir, elbasvir, and ombitasvir) have demonstrated potential interactions with a range of drug-metabolizing enzymes and transporters (Kiser et al., 2013; http://www.natap.org/2016/Pharm/Pharm_54. htm). In this paper, we characterized the potential for the combination of glecaprevir and pibrentasvir to increase exposure of the substrates for clinically relevant transporters.

In vitro inhibition of P-gp, BCRP, OATP1B1/3, OCT1/2, OAT1/3, and MATE1/2K was evaluated for glecaprevir and pibrentasvir. Owing to the high plasma protein binding (>99.9\%) and poor solubility of pibrentasvir, an additional inhibition assessment was conducted for OATP1B1/3, OCT1/ 2, OAT1/3, and MATE1/2K in the presence of the physiologically relevant concentration of albumin, i.e., the total $\mathrm{IC}_{50}$ method (Kikuchi et al., 2017). Glecaprevir was identified as an inhibitor of P-gp, BCRP, OATP1B1, and OATP1B3, while pibrentasvir was an inhibitor for P-gp, BCRP, and OATP1B1. Glecaprevir and pibrentasvir did not inhibit OCT1, OCT2, OAT1, OAT3, MATE1, and MATE2K.

Consistent with the anticipated inhibition of intestinal P-gp, the combination of glecaprevir and pibrentasvir increased the bioavailability of the P-gp substrates digoxin and dabigatran etexilate. Intestinal P-gp governs drug-drug interactions with many common orally administered drugs and the magnitude of interactions may depend on both the absolute bioavailability of individual substrates and the extent of P-gp inhibition (Greiner et al., 1999; Härtter et al., 2013; Pradaxa (dabigatran etexilate mesylate capsules), 2015). Digoxin exhibits $60 \%-80 \%$ absolute bioavailability when administered as a tablet, is predominantly eliminated 
by renal excretion, and does not undergo significant enterohepatic circulation (Marcus et al., 1976; Lloyd et al., 1978). Thus, the digoxin AUC may only be increased a maximum of $25 \%-67 \%$ by inhibition of intestinal efflux, which affects bioavailability, not clearance-increases up to $150 \%$ have been reported upon coadministration with dronedarone and likely reflect additive inhibition of digoxin renal tubular secretion (Hori et al., 1993; Vallakati et al., 2013). The $48 \%$ increase in digoxin AUC observed with glecaprevir and pibrentasvir and accompanying modest impact on digoxin terminal elimination half-life in plasma are suggestive, but not conclusive, of an intestinal-based interaction. The prodrug dabigatran etexilate is a $\mathrm{P}$-gp substrate, but it is rapidly converted to dabigatran, which is not transported by $\mathrm{P}$-gp (Stangier and Clemens, 2009). Changes in dabigatran exposure following coadministration of dabigatran etexilate with P-gp inhibitors are thus restricted to interactions at the level of the intestinal epithelium, making it an ideal selective probe for intestinal P-gp inhibition. The low oral bioavailability of dabigatran etexilate also confers high sensitivity; dabigatran AUC increases up to $15 \%$ have been reported with the P-gp inhibitors ketoconazole and dronedarone (Pradaxa (dabigatran etexilate mesylate capsules), 2013; 2015; Zhao and $\mathrm{Hu}$, 2014). Despite lower sensitivity, interactions of digoxin with $\mathrm{P}$-gp inhibitors remain clinically relevant given the narrow therapeutic index of the drug (Liu et al., 2017).

As a drug class, statins are prototypical examples of clinically relevant OATP1B1/3 interactions since increased statin exposures are associated with the risk of myopathy or rhabdomyolysis (Giacomini et al., 2010). The magnitude of clinical statin exposure increases depends on the sensitivity of individual statins toward inhibition of OATP1B1/3-mediated hepatic uptake; however, many statins are also substrates of other drug-metabolizing enzymes or transporters that may also be inhibited by a concomitant medication (e.g., CYP3A, P-gp, and BCRP), making extrapolation among different statin drugs difficult. In vitro data suggest that glecaprevir and pibrentasvir may inhibit OATP1B1 and/or OATP1B3 (Table 1). In line with these projections, the glecaprevir and pibrentasvir combination increased clinical exposures of pravastatin and rosuvastatin. When evaluating the effect of genetic polymorphisms on statins, a reduced OATP1B1 function phenotype was associated with similar increases in AUC for pravastatin and rosuvastatin (61\%-91\%), but a reduced BCRP function phenotype was associated with higher rosuvastatin AUC increases (144\%) than pravastatin (13\%) (Kellick et al., 2014). Glecaprevir and pibrentasvir were identified as BCRP inhibitors in vitro, and the observed increases in rosuvastatin exposure may result from the contribution of both OATP1B1/3 and BCRP inhibition. It should be noted that genetic polymorphisms in BCRP may impact transporter expression and/or function throughout various tissues, and in the case of rosuvastatin may reduce active renal secretion (Verhulst et al., 2008). In contrast, clinically relevant inhibition of BCRP by glecaprevir and pibrentasvir is confined to the gut since systemic concentrations are insufficient to inhibit this transporter in other tissues, such as kidney.

Sofosbuvir is a substrate of P-gp and BCRP, but its predominant metabolite GS-331007 is not. GS-331007 does not exhibit anti-HCV activity, but concentrations are reflective of the pharmacologically active intracellular triphosphate sofosbuvir metabolite. Systemic sofosbuvir concentrations may increase as a result of P-gp and/or BCRP inhibition without concurrent impact on GS-331007, and as per prescribing information sofosbuvir can be administered concomitantly with P-gp and BCRP inhibitors in a clinical setting (Kirby et al., 2015; Sovaldi (sofosbuvir), 2017). Thus, changes in sofosbuvir exposure are useful in confirming DDI mechanisms identified with other probe substrates, but are not expected to result in clinically significant interactions.

The selected substrates have limited potential to act as perpetrators of drug-drug interactions and were not expected to affect glecaprevir or pibrentasvir. Glecaprevir exposures were $44 \%-59 \%$ higher in the presence of pravastatin, but these increases are not considered clinically relevant. None of the other probe substrates evaluated here affected exposures of glecaprevir or pibrentasvir.

The Food and Drug Administration recommends that for clinical DDI studies, potential perpetrator drugs are administered at the highest approved dose and shortest dosing interval (https://www.fda.gov/downloads/drugs/guidances/ ucm292362.pdf). Most of the clinical DDI studies presented here were conducted with glecaprevir $400 \mathrm{mg}$, a larger dose than the $300 \mathrm{mg}$ used as part of the approved glecaprevir/ pibrentasvir clinical regimen. In vitro projections were based on clinical exposures at the $300 \mathrm{mg}$ dose level and suggest that transport inhibition remains relevant for the clinical regimen. When coadministered with glecaprevir, pibrentasvir exposures are higher than for pibrentasvir alone in a mechanism attributed to inhibition of intestinal P-gp and/or BCRP by glecaprevir. These increases were similar for glecaprevir 200-400 mg doses, increasing the pibrentasvir AUC to 3.0to 3.5-fold and suggesting a saturation of this inhibition in the clinical dose range (Lin et al., 2017, 2018); potent inhibition of intestinal P-gp with glecaprevir $300 \mathrm{mg}$ was confirmed in the dabigatran etexilate study. Similarly, substantial OATP1B1/3 interactions for glecaprevir $300 \mathrm{mg}$ were observed in subsequent studies with simvastatin and lovastatin (Mavyret, 2019). No clinical drug-drug interaction studies with sensitive substrates of BCRP were conducted at a dose of glecaprevir $300 \mathrm{mg}$, but $I_{\text {gut }} / \mathrm{IC}_{50}$ values calculated for glecaprevir and pibrentasvir based on exposures for the approved 300/120 mg regimen remain several fold above the threshold for potential clinical relevance (https://www.fda.gov/downloads/Drugs/ Guidances/UCM581965.pdf). For victim drugs where a potential increase in exposure is anticipated, utilization of the highest clinical dose in clinical DDI studies may be inadvisable. Dabigatran etexilate, digoxin, pravastatin, rosuvastatin, and sofosbuvir all exhibit linear pharmacokinetics at the study doses, in line with the Food and Drug Administration recommendations (https://www.fda.gov/downloads/drugs/ guidances/ucm292362.pdf). Thus, the studies presented here are appropriate for informing clinical dosing guidance.

In conclusion, predictions based on in vitro results indicated the potential for clinically relevant inhibition of P-gp, BCRP, and OATP1B1/3 transporters by the glecaprevir and pibrentasvir combination. These predictions were confirmed in a clinical setting where the combination regimen increased exposure of probe transporter substrates. Health care providers should refer to glecaprevir/pibrentasvir prescribing information for appropriate dosing recommendations on concomitant use with these and other transport substrates in patients. 


\section{Acknowledgments}

The authors thank the clinical sites and investigators and AbbVie biometrics statisticians Bifeng Ding, Hong Li, and Weihan Zhao; AbbVie clinical investigators David Carter, Ken Kamradt, and Dilraj Sidhu; AbbVie study-designated physicians Armen Asatryan, David Pugatch, Joaquin Valdes, and Stanley Wang; AbbVie drug metabolism and pharmacokinetics scientists Zhizhou Fang, William Chiou, Vincent Peterkin, and bioanalysis support personnel; and Amy Rohrlack, an AbbVie employee, for medical writing support.

\section{Authorship Contributions}

Participated in research design: Kosloski, Bow, Kikuchi, Wang, Mensa, Kort, Liu.

Performed data analysis: Kosloski, Bow, Kikuchi, Wang, Liu, Kim, Marsh.

Wrote or contributed to the writing of the manuscript: Kosloski, Bow, Kikuchi, Wang, Mensa, Kort, Liu, Kim, Marsh.

\section{References}

Back D and Else L (2013) The importance of drug-drug interactions in the DAA era. Dig Liver Dis 45 (Suppl 5):S343-S348.

Bourlière $\mathrm{M}$ and Pietri $\mathrm{O}$ (2019) Hepatitis $\mathrm{C}$ virus therapy: no one will be left behind Int $J$ Antimicrob Agents 53:755-760.

Chu X, Galetin A, Zamek-Gliszczynski MJ, Zhang L, and Tweedie DJ; International Transporter Consortium (2018) Dabigatran etexilate and digoxin: comparison as clinical probe substrates for evaluation of P-gp inhibition. Clin Pharmacol Ther 104:788-792.

Elsby R, Hilgendorf C, and Fenner K (2012) Understanding the critical disposition pathways of statins to assess drug-drug interaction risk during drug development: it's not just about OATP1B1. Clin Pharmacol Ther 92:584-598.

Gane E, Lawitz E, Pugatch D, Papatheodoridis G, Bräu N, Brown A, Pol S, Leroy V, Persico M, Moreno C, et al. (2017) Glecaprevir and pibrentasvir in patients with HCV and severe renal impairment. N Engl J Med 377:1448-1455.

Giacomini KM, Huang SM, Tweedie DJ, Benet LZ, Brouwer KL, Chu X, Dahlin A, Evers R, Fischer V, Hillgren KM, et al.; International Transporter Consortium (2010) Membrane transporters in drug development. Nat Rev Drug Discov 9: $215-236$.

Greiner B, Eichelbaum M, Fritz P, Kreichgauer HP, von Richter O, Zundler J, and Kroemer HK (1999) The role of intestinal P-glycoprotein in the interaction of digoxin and rifampin. $J$ Clin Invest 104:147-153.

Härtter S, Koenen-Bergmann M, Sharma A, Nehmiz G, Lemke U, Timmer W, and Reilly PA (2012) Decrease in the oral bioavailability of dabigatran etexilate after co-medication with rifampicin. Br J Clin Pharmacol 74:490-500.

Härtter S, Sennewald R, Nehmiz G, and Reilly P (2013) Oral bioavailability of dabigatran etexilate (Pradaxa®) after co-medication with verapamil in healthy subjects. Br J Clin Pharmacol 75:1053-1062.

Höner Zu Siederdissen C, Maasoumy B, Marra F, Deterding K, Port K, Manns MP, Cornberg M, Back D, and Wedemeyer H (2016) Drug-drug interactions with novel all oral interferon-free antiviral agents in a large real-world cohort. Clin Infect Dis 62:561-567.

Hori R, Okamura N, Aiba T, and Tanigawara Y (1993) Role of P-glycoprotein in renal tubular secretion of digoxin in the isolated perfused rat kidney. J Pharmacol Exp Ther 266:1620-1625.

Kellick KA, Bottorff M, and Toth PP; The National Lipid Association's Safety Task Force (2014) A clinician's guide to statin drug-drug interactions. J Clin Lipidol 8 (Suppl 3):S30-S46.
Kikuchi R, Peterkin VC, Chiou WJ, de Morais SM, and Bow DAJ (2017) Validation of a total $\mathrm{IC}_{50}$ method which enables in vitro assessment of transporter inhibition under semi-physiological conditions. Xenobiotica 47:825-832.

Kirby BJ, Symonds WT, Kearney BP, and Mathias AA (2015) Pharmacokinetic, pharmacodynamic, and drug-interaction profile of the hepatitis $\mathrm{C}$ virus NS5B polymerase inhibitor sofosbuvir. Clin Pharmacokinet 54:677-690.

Kiser JJ, Burton JR Jr, and Everson GT (2013) Drug-drug interactions during antiviral therapy for chronic hepatitis C. Nat Rev Gastroenterol Hepatol 10:596-606. Lin CW, Dutta S, Asatryan A, Wang H, Clifton J II, Campbell A, and Liu W (2018) Pharmacokinetics, safety, and tolerability following single and multiple doses of pibrentasvir in a first-in-human study. Clin Pharmacol Drug Dev 7:44-52.

Lin CW, Dutta S, Ding B, Wang T, Zadeikis N, Asatryan A, Kort J, Campbell A Podsadecki T, and Liu W (2017) Pharmacokinetics, safety, and tolerability of glecaprevir and pibrentasvir in healthy White, Chinese, and Japanese adult subjects. $J$ Clin Pharmacol 57:1616-1624.

Liu H, Michmerhuizen MJ, Lao Y, Wan K, Salem AH, Sawicki J, Serby M, Vaidyanathan S, Wong SL, Agarwal S, et al. (2017) Metabolism and disposition of a novel B-cell lymphoma-2 inhibitor venetoclax in humans and characterization of its unusual metabolites. Drug Metab Dispos 45:294-305.

Lloyd BL, Greenblatt DJ, Allen MD, Harmatz JS, and Smith TW (1978) Pharmacokinetics and bioavailability of digoxin capsules, solution and tablets after single and multiple doses. Am J Cardiol 42:129-136.

Louie KS, St Laurent S, Forssen UM, Mundy LM, and Pimenta JM (2012) The high comorbidity burden of the hepatitis $\mathrm{C}$ virus infected population in the United States. BMC Infect Dis 12:86.

Marcus FI, Dickerson J, Pippin S, Stafford M, and Bressler R (1976) Digoxin bioavailability: formulations and rates of infusions. Clin Pharmacol Ther 20:253-259.

Mavyret (glecaprevir and pibrentasvir tablets). (2019) Package insert. AbbVie Inc., North Chicago, IL.

Ng TI, Krishnan P, Pilot-Matias T, Kati W, Schnell G, Beyer J, Reisch T, Lu L, Dekhtyar T, Irvin M, et al. (2017) In vitro antiviral activity and resistance profile of the next-generation hepatitis C virus NS5A inhibitor pibrentasvir. Antimicrob Agents Chemother 61:e02558-16.

Ng TI, Tripathi R, Reisch T, Lu L, Middleton T, Hopkins TA, Pithawalla R, Irvin M Dekhtyar T, Krishnan P, et al. (2018) In vitro antiviral activity and resistance profile of the next-generation hepatitis $\mathrm{C}$ virus NS3/4a protease inhibitor glecaprevir. Antimicrob Agents Chemother 62:e01620-17.

Pradaxa (dabigatran etexilate mesylate capsules). (2013) Summary of product characteristics. Boehringer Ingelheim International GmbH, Ingelheim am Rhein, Germany.

Pradaxa (dabigatran etexilate mesylate capsules). (2015) Package insert. Boehringer Ingelheim Pharmaceuticals Inc., Ridgefield, CT.

Sovaldi (sofosbuvir). (2017) Package insert. Gilead Sciences Inc., Foster City, CA.

Serfaty L (2013) Optimizing DAA management in daily practice. Dig Liver Dis 45 (Suppl 5):S318-S322.

Stangier J and Clemens A (2009) Pharmacology, pharmacokinetics, and pharmacodynamics of dabigatran etexilate, an oral direct thrombin inhibitor. Clin Appl Thromb Hemost 15 (Suppl 1):9S-16S.

Vallakati A, Chandra PA, Pednekar M, Frankel R, and Shani J (2013) Dronedaroneinduced digoxin toxicity: new drug, new interactions. Am J Ther 20:e717-e719.

Verhulst A, Sayer R, De Broe ME, D'Haese PC, and Brown CD (2008) Human proximal tubular epithelium actively secretes but does not retain rosuvastatin. $\mathrm{Mol}$ Pharmacol 74:1084-1091.

Zhao Y and Hu ZY (2014) Physiologically based pharmacokinetic modelling and in vivo $[\mathrm{I}] / K_{\mathrm{i}}$ accurately predict P-glycoprotein-mediated drug-drug interactions with dabigatran etexilate. Br J Pharmacol 171:1043-1053.

Address correspondence to: Wei Liu, AbbVie, Inc., Clinical Pharmacokinetics and Pharmacodynamics, Department R4PK, Building AP31-3, 1 North Waukegan Road, North Chicago, IL, 60064. E-mail: wei.liu@abbvie.com 